\title{
Optimal Flight for Ground Noise Reduction in Helicopter Landing Approach: Optimal Altitude and Velocity Control
}

\author{
By Takeshi TsuchiYa, ${ }^{1)}$ Hirokazu IsHII, ${ }^{2)}$ Junichi UCHIDA, ${ }^{1)}$ Hiromi Gomi, ${ }^{2)}$ \\ Naoki MATAYOSHI ${ }^{2)}$ and Yoshinori OKUNO ${ }^{2)}$ \\ ${ }^{1)}$ Department of Aeronautics and Astronautics, The University of Tokyo, Tokyo, Japan \\ ${ }^{2)}$ Aviation Program Group, Japan Aerospace Exploration Agency, Tokyo, Japan
}

(Received December 25th, 2006)

\begin{abstract}
This study aims to obtain the optimal flights of a helicopter that reduce ground noise during landing approach with an optimization technique, and to conduct flight tests for confirming the effectiveness of the optimal solutions. Past experiments of Japan Aerospace Exploration Agency (JAXA) show that the noise of a helicopter varies significantly according to its flight conditions, especially depending on the flight path angle. We therefore build a simple noise model for a helicopter, in which the level of the noise generated from a point sound source is a function only of the flight path angle. Using equations of motion for flight in a vertical plane, we define optimal control problems for minimizing noise levels measured at points on the ground surface, and obtain optimal controls for specified initial altitudes, flight constraints, and wind conditions. The obtained optimal flights avoid the flight path angle which generates large noise and decrease the flight time, which are different from conventional flight. Finally, we verify the validity of the optimal flight patterns through flight experiments. The actual flights following the optimal paths resulted in noise reduction, which shows the effectiveness of the optimization.
\end{abstract}

Key Words: Guidance and Control, Helicopter, Noise Abatement, Optimal Control

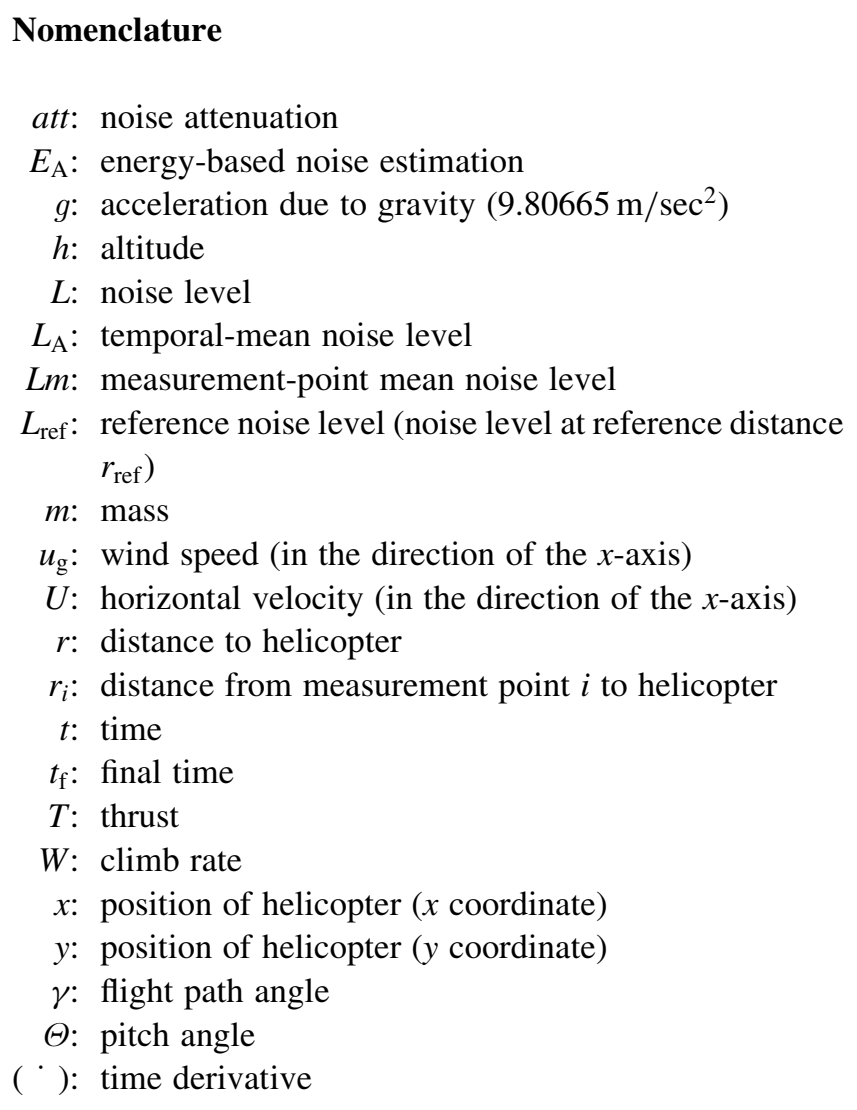

\section{Introduction}

Helicopters, which are different from fixed-wing aircraft

(C) 2007 The Japan Society for Aeronautical and Space Sciences in that helicopters do not require a large runway during takeoff and landing and have high maneuverability, are relied upon as effective means of transport in suburban and mountainous areas. However, at present they are utilized only for missions such as reporting or disaster and rescue emergencies. One reason for this is that a helicopter poses the problem of noise. A large amount of noise in the environment surrounding their flight paths is arguably one of the main reasons preventing their more widespread use. While noise originates throughout a helicopter, (e.g., rotor, engine and transmission), a particular noise occurs during takeoff, landing and flight at low altitude, which is known as blade vortex interaction (BVI) noise. BVI noise occurs when wingtip vortexes emitted from the main rotor blades interfere with the rear blades. Since the magnitude, which varies with flight conditions, is particularly strong during descent, landing approach at low altitude produces a large amount of noise damage to the environment. Meanwhile, since the selection of a flight path is basically entrusted to a pilot's judgment, transportation in densely populated areas depends on the experience of the pilot who should take into consideration the noise affecting the surrounding area. However, since propagation characteristics of helicopter noise change due to factors such as climate conditions, the actual noise at ground level is not necessarily consistent with the noise experienced by the pilot. In particular, it is possible that flight in areas where the pilot does not know the landscape inflicts serious noise damage. Thus, in the future, it will be necessary to reduce the damage by dispersing the noise over tolerant areas such as rivers, commercial regions and forests using a pre- 


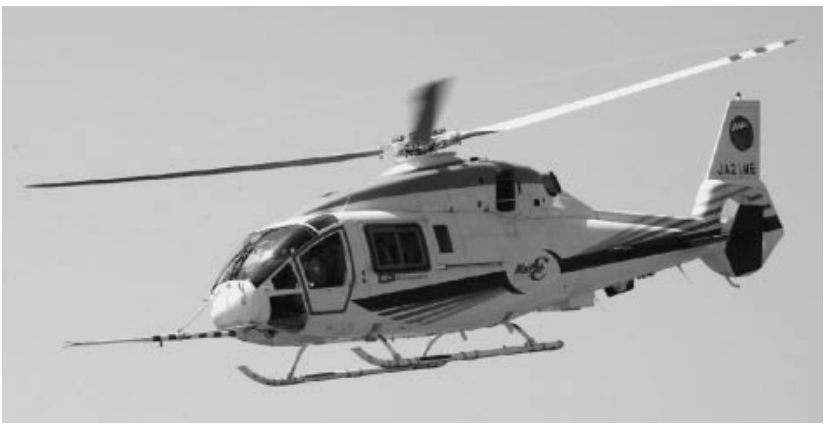

Fig. 1. The JAXA multipurpose experimental helicopter, MuPAL- $\varepsilon$.

cision approach system, ${ }^{1)}$ one of which is developed by the Japan Aerospace Exploration Agency (JAXA).

Based on the background described above, JAXA is developing a system to estimate and display ground-level noise damage due to a helicopter in real time on board the vehicle. ${ }^{2)}$ They have advanced research on methods for measuring noise using actual aircraft, and measured the noise at ground level and on board an experimental helicopter, MuPAL- $\varepsilon$ (Fig. 1). ${ }^{3,4)}$ They have also calculated rotor noise using computational fluid dynamics (CFD). The computational accuracy of CFD has increased with advances in computational techniques and computing power, and CFD has been applied to studies on noise reduction through improvements in blade shape. ${ }^{5)}$ However, it is not possible to estimate noise damage for actual flights in real time with the current computing power. In contrast, the first author of this paper has studied numerical solution methods of optimal control problems at the University of Tokyo. The study aims at an optimization algorithm, which is applicable to problems close to actual existing conditions, with relatively high versatility. ${ }^{6}$ With this background, JAXA and the University of Tokyo are currently pursuing collaborative research regarding landing approach trajectories that minimize helicopter noise damage. This study focuses on the fact that the noise emanating from a helicopter depends on flight conditions, and aims to reduce the ground-level noise damage by optimizing the landing approach flight path using numerical computation methods.

Overseas researches related to numerical models of helicopter and aircraft noise are advancing at the FAA, NASA and universities in the United States. In Europe, the EU research project, SILENCER, is developing noise-reduced aircraft. The Integrated Noise Model (INM) developed by the FAA, which uses a simple model expressing aircraft noise as a point sound source, is in broad use at flight operation sites around the world. NASA established the Rotorcraft Noise Model $(\mathrm{RNM})^{7)}$ that models sound sources three-dimensionally, but as yet no examples of its application to the optimization of an actual flight path have been presented.

Building on the above, the aim of the present research is to find flight trajectories that minimize ground-level noise during helicopter landing approaches using computational optimization. The main objective of this paper is to verify the validity of various models, such as a noise model and helicopter motion model. For the sake of simplicity, the helicopter's motion in only the vertical plane (i.e., altitude and velocity control) is considered, which means that we do not deal with horizontal control herein. A further objective is to confirm, during flight tests using an actual helicopter, effective noise abatement on flight paths based on the optimal solutions. This paper first outlines the flight test environment, the helicopter used, and flight conditions. Optimal control problems are then defined accordingly and the results of computational optimization are shown. Thereafter, we present the results of the flight tests. Finally, we explain future research.

\section{Summary of Optimally Controlled Flights}

In preparation for the definition of optimal control problems in the next section, this section summarizes flight tests in which we experimentally validate the results of the optimal controls. The optimal control problems are defined according to the flight test environment.

We carried out flight tests in the vicinity of Ryugasaki Airfield in Ibaraki Prefecture using an experimental helicopter, MuPAL- $\varepsilon$, owned by JAXA. The airspace above Ryugasaki Airfield has an upper altitude limit because it is under special control by Narita Airport. We had to consider this upper limit and other issues such as the avoidance of areas sensitive to noise damage and the measurement of ground-level noise at points far from roads with heavy traffic. We installed five noise measurement points on the ground directly below an approach path straight towards the runway of Ryugasaki Airfield. In this paper, the helicopter's position is expressed using a coordinate frame with a target point of the approach (a supposed landing point) as the origin, the flight direction as the $+x$ axis, and the right-hand side with respect to the flight direction as the $+y$ axis. Table 1 shows the coordinates of the noise measurement points. The noise measurement points are numbered from 1 to 5 in order of increasing distance from the target point. The coordinates of the helicopter's initial point at the moment it begins the landing approach are set to $(x, y)=(-3.0,0)[\mathrm{NM}](=(-5.56,0)[\mathrm{km}])$, and the altitude at that time is approximately $450-600 \mathrm{~m}$. The coordinates of the terminal point are $(x, y)=(-0.47,0)[\mathrm{NM}](=(-870,0)$ $[\mathrm{m}])$, with an altitude of $300 \mathrm{ft}(91.4 \mathrm{~m})$. In order for the helicopter to pass through the airspace above the measurement points from the initial point to the terminal point, only the altitude and velocity are controlled during flight. That is to

Table 1. Coordinates of noise measurement points.

\begin{tabular}{crr}
\hline Noise measurement point & $x[\mathrm{~km}]$ & $y[\mathrm{~km}]$ \\
\hline 1 & -5.54 & 0.00 \\
2 & -4.04 & 0.11 \\
3 & -3.41 & 0.04 \\
4 & -2.17 & -0.02 \\
5 & -1.35 & 0.00 \\
\hline
\end{tabular}


say, the helicopter does not perform sideways motion such as roll motion. The aim of this study is to find a flight pattern minimizing the noise levels measured at the five measurement points. Since final maneuvering from the terminal point at an altitude of $300 \mathrm{ft}$ to landing on the ground is left to a pilot of the helicopter, it is outside the scope of the optimal controls in this paper.

\section{Computational Optimal Control}

\subsection{Definition of the optimal control problems}

To derive helicopter equations of motion in this paper, the helicopter is treated as a point mass, and its motion is limited to the vertical plane. According to these assumptions, the equations of motion are as follows.

$$
\begin{aligned}
\dot{x} & =U+u_{\mathrm{g}} \\
\dot{h} & =W \\
\dot{U} & =-\frac{T}{m} \sin \Theta \\
\dot{W} & =g-\frac{T}{m} \cos \Theta
\end{aligned}
$$

There are two control parameters: the thrust, $T$ and pitch angle, $\Theta$. These equations of motion include only two external forces acting on the helicopter: the thrust of the main rotor and gravity. Aerodynamic forces are ignored. The wind acting on the helicopter $\left(u_{\mathrm{g}}\right)$ is assumed to be purely horizontal and constant, regardless of altitude or time. We substitute possible wind speeds in the test environment for $u_{\mathrm{g}}$. The specific values substituted are discussed later.

Next, we explain the initial and terminal conditions of the optimal control problems. The initial conditions at $t=0$ are related to the state when the landing approach begins, which is explained in the previous section.

$$
\begin{aligned}
& x(0)=-3.0[\mathrm{NM}](-5.56[\mathrm{~km}]) \\
& h(0)=1500[\mathrm{ft}](457[\mathrm{~m}]) \text { or } 1850[\mathrm{ft}](564[\mathrm{~m}]) \\
& U(0)=100[\mathrm{kt}](51.4[\mathrm{~m} / \mathrm{sec}]) \\
& W(0)=0[\mathrm{fpm}]
\end{aligned}
$$

When the flight begins, the helicopter flies horizontally and the airspeed is set to $100 \mathrm{kt}$. We consider two cases of the initial conditions, of which altitudes are $1500 \mathrm{ft}$ and $1850 \mathrm{ft}$. The reason for defining these two initial altitudes is discussed later. The terminal conditions at $t=t_{\mathrm{f}}$ indicate completion of the landing approach flights. As described in the previous section, we define as follows.

$$
\begin{aligned}
& x\left(t_{\mathrm{f}}\right)=-0.47[\mathrm{NM}](-870[\mathrm{~m}]) \\
& h\left(t_{\mathrm{f}}\right)=300[\mathrm{ft}](91.4[\mathrm{~m}]) \\
& U\left(t_{\mathrm{f}}\right)=50[\mathrm{kt}](25.7[\mathrm{~m} / \mathrm{sec}]) \\
& W\left(t_{\mathrm{f}}\right)=-500[\mathrm{fpm}](-2.54[\mathrm{~m} / \mathrm{sec}])
\end{aligned}
$$

The final time, $t_{\mathrm{f}}$, is also an optimized parameter.

In addition, we define constraint conditions during flight. The helicopter motion is calculated with the above-men- tioned simple model, but the actual helicopter is controlled by the pilot manually in the flight experiments. It is therefore necessary for the pilot to follow the optimal solutions without being burdened by an excessive workload. We made an experiment of a flight simulator owned by JAXA to determine the constraint conditions, and then settled upon three types of constraint conditions listed below.

- Horizontal-velocity limit

$$
50 \leq U \leq 100[\mathrm{kt}](25.7 \leq U \leq 51.4[\mathrm{~m} / \mathrm{sec}])
$$

- Climb rate limit

Considering the psychological impact on the pilot taking a steep dive at low altitude, we differentiate constraint conditions by altitude in order to establish a relatively strict limit below $600 \mathrm{ft}(183 \mathrm{~m})$.

When $h \geq 600 \mathrm{ft}$,

$$
|W| \leq 1000[\mathrm{fpm}](5.08[\mathrm{~m}]) .
$$

When $h \leq 600 \mathrm{ft}$,

$$
-\frac{5}{3} h \leq W \leq 1000[\mathrm{fpm}] .
$$

In order for the helicopter to descend consistently in landing approach, we also solve the problem where the upper limit on the climb rate is replaced with

$$
W \leq 0 \text {. }
$$

The details are discussed later.

- Acceleration limit

$$
\begin{aligned}
& |\dot{U}| \leq 1.5[\mathrm{kt} / \mathrm{sec}]\left(0.771\left[\mathrm{~m} / \mathrm{sec}^{2}\right]\right) \\
& |\dot{W}| \leq 100[\mathrm{fpm} / \mathrm{sec}]\left(0.508\left[\mathrm{~m} / \mathrm{sec}^{2}\right]\right)
\end{aligned}
$$

\subsection{Sound-source model and attenuation model}

Helicopter noise emanates from various sources such as the rotor, engine and gearbox. In addition, the frequency and directivity depend on the flight conditions. However, considering the computational load of optimization, this study first uses a simplified sound source model ignoring the directivity. Figure 2 plots the relationship between airspeed, flight path angle and noise level measured using a helicopter-mounted microphone attached to the nose of $\mathrm{Mu}-$ PAL $-\varepsilon{ }^{8)}$ The noise levels given hereafter compensate for human hearing using A-weighting. Figure 2 indicates the noise levels converted to the levels measured at a distance of $100 \mathrm{~m}$ from the helicopter (reference distance $r_{\text {ref }}$ ). The results show that the noise of the helicopter (MuPAL- $\varepsilon$ ) strongly depends on the flight path angle $\gamma$ (= $\tan ^{-1} W / U$ ), but the difference in noise due to the airspeed is not large. The noise is largest around a flight path angle of $-5 \mathrm{deg}$. The cause is BVI noise. To incorporate the characteristics of these results, the helicopter is assumed to have a sound source with a simplified reference noise level $L_{\text {ref }}$ [dB] at reference distance $r_{\text {ref }}=100[\mathrm{~m}]$ (the curve in Fig. 2).

- when $\gamma<-15[\mathrm{deg}]$ or $5[\mathrm{deg}]<\gamma$,

$$
L_{\mathrm{ref}}(\gamma)=80 \text {. }
$$




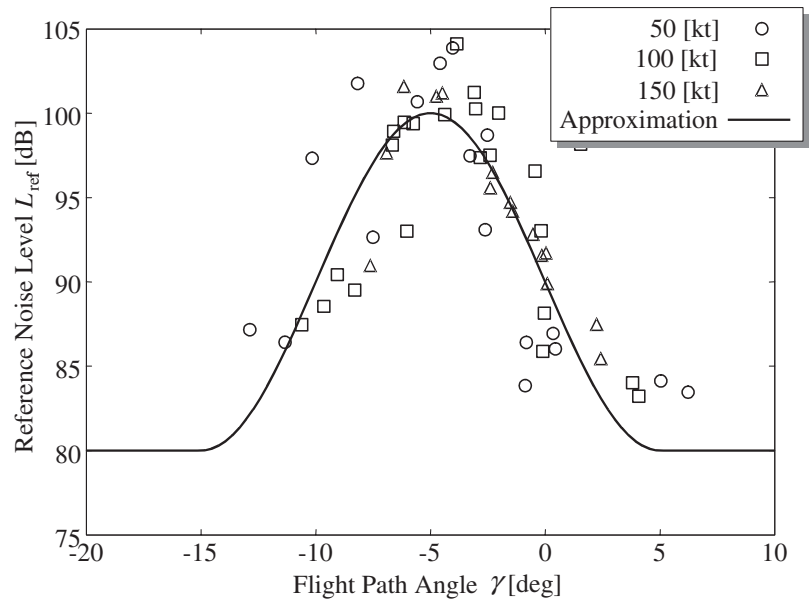

Fig. 2. Relationship between flight speed, flight path angle and noise level.

- when $-15[\mathrm{deg}] \leq \gamma \leq 5[\mathrm{deg}]$,

$$
L_{\mathrm{ref}}(\gamma)=90+10 \cos \left(\pi \frac{\gamma+5}{10}\right) .
$$

The unit of the flight path angle $\gamma$ in Eq. (20) is degrees.

The noise emitted from the helicopter is subject to the influence of various factors during its propagation to ground level, such as the inverse square law of distance, atmospheric damping, ground-surface damping, wind, air temperature, and humidity. This study considers only the inverse square law as a model of attenuation. That is to say, the attenuation $\operatorname{att}(r)[\mathrm{dB}]$ at a point with distance $r$ from the sound source is

$$
\operatorname{att}(r)=20 \log \frac{r}{r_{\text {ref }}} .
$$

According to Eqs. (19), (20) and (21), at time $t$, the noise level $L(t)[\mathrm{dB}]$ measured at a distance $r(t)$ from the helicopter flying at a flight path angle $\gamma(t)$ is calculated as

$$
L(t)=L_{\mathrm{ref}}(\gamma(t))-\operatorname{att}(r(t)) .
$$

In addition, this study ignores the time for the noise to propagate from the sound source to the measurement point. Comparing the results of the computational optimization with flight test results, we will confirm the validity of the proposed sound source model and attenuation model.

\subsection{Performance index}

While the level of the helicopter noise measured at a point on the ground is estimated objectively as described in the previous section, it is a subjective problem as to how unpleasant the experience is for a person. That is to say, noise estimation must reflect the degree of influence on human psychology and physiology, but on the other hand it must be able to be measured objectively. In this study therefore, an energy-based noise estimation is used as a noise estimation index, by making reference to noise exposure level, ${ }^{9}$ which is widely used for aircraft noise estimation. This is computed from time-integrating the energy of the noise level occurring at each instant. Since there are multiple measurement points in this study, the noise level is taken to be the algebraic mean of the noise levels measured at each measurement point. That is, supposing measurement point $i(i=1,2,3,4,5)$ is at distance $r_{i}(t)$ from the helicopter at time $t$, the measurement-point mean noise level $\operatorname{Lm}(t)$ $[\mathrm{dB}]$ at time $t$ is calculated as follows.

$$
\operatorname{Lm}(t)=\frac{1}{5} \sum_{i=1}^{5}\left[L_{\mathrm{ref}}(\gamma(t))-\operatorname{att}\left(r_{i}(t)\right)\right]
$$

The energy-based noise estimation $E_{\mathrm{A}}$, which is the performance index of the optimal control problem, is calculated by converting this measurement-point mean noise level $\operatorname{Lm}(t)$ into energy, and taking the time integral over the entire flight time.

$$
E_{\mathrm{A}}=\int_{0}^{t_{\mathrm{f}}} 10^{\frac{L m(t)}{10}} \mathrm{~d} t
$$

Minimizing this index is the objective of the optimal control.

\subsection{Three cases of computational optimization}

For the problem defined above, the constraint conditions are set according to the three cases stated below, and computational optimization is performed.

Case 1: The initial altitude $h(0)=1500$ [ft].

Case 2: The initial altitude $h(0)=1500$ [ft]. In order to avoid ascent during flight, the upper limit on the climb rate is changed $(W \leq 0)$.

Case 3: The initial altitude $h(0)=1850$ [ft]. In order to avoid ascent during flight, the upper limit on the climb rate is changed.

The reason for changing the constraint conditions in Cases 2 and 3 is because we foresaw that the suppression of ground-level noise while flying (i.e., Case 1) would result in an optimal solution in which the helicopter ascended and flew at a high altitude. Thus, we constrain the climb rate in order to obtain a flight close to a conventional landing approach. In addition, the reason for setting the initial altitude to $1850 \mathrm{ft}$ in Case 3 is due to the optimal solution for Case 1 . This will be discussed later. Moreover, for each of these three cases, the optimal solutions are computed by substituting a value between $-20 \mathrm{kt}(-10.3 \mathrm{~m} / \mathrm{sec})$ and $20 \mathrm{kt}(10.3$ $\mathrm{m} / \mathrm{sec}$ ) for the wind speed $u_{\mathrm{g}}$.

Computational optimization is conducted with the helicopter model, constraint conditions, sound source model, propagation model and performance index described above. Computational optimization is performed using the $\mathrm{BDH}$ method, ${ }^{6}$ which can incorporate all kinds of constraint conditions and excels in convergence characteristic. This method discretizes all of the state variables, control variables, motion equations and constraint condition equations, which are functions of time, and transforms the optimal control problem, which is a dynamic-optimization problem, into a nonlinear programming problem that is a static-optimization problem. An optimal solution to the converted nonlinear programming problem is computed using a sequential quadratic programming (SQP) method, which is a class of gradient method. 


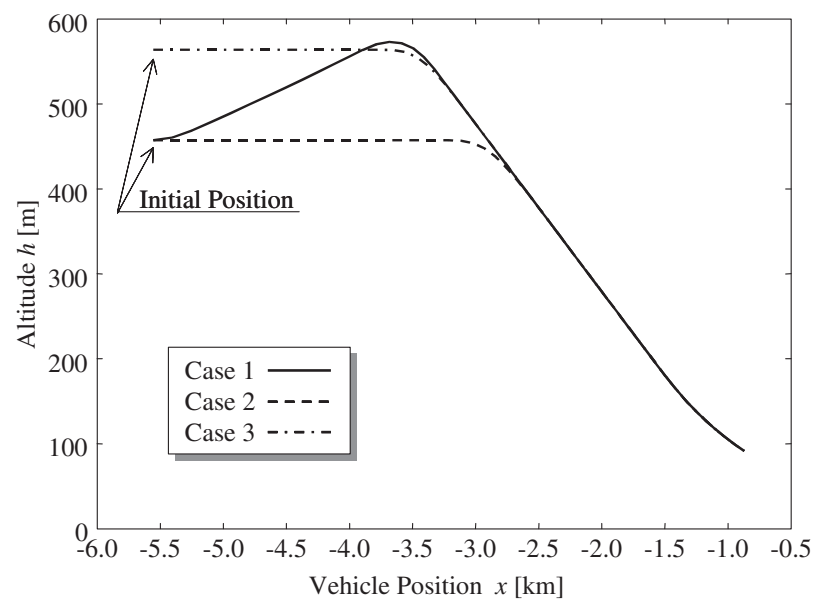

Fig. 3. Optimal solutions (altitude history).

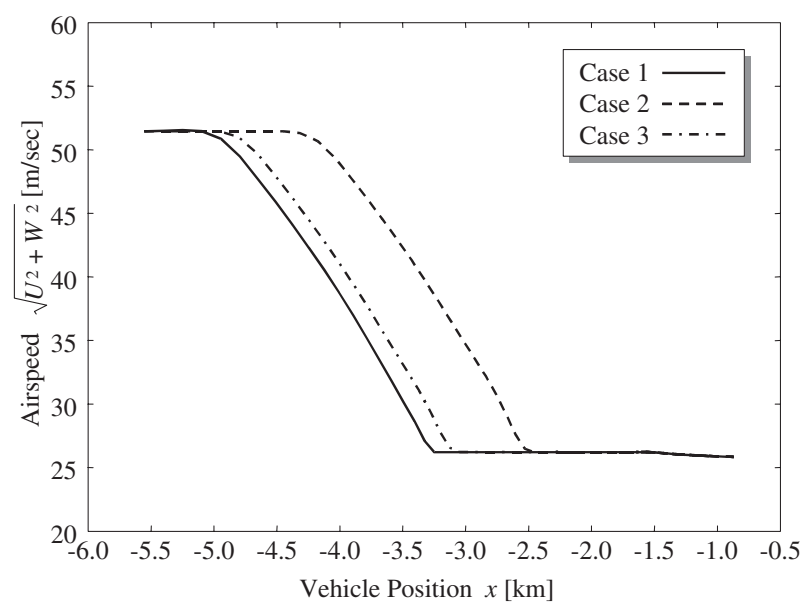

Fig. 4. Optimal solutions (airspeed history).

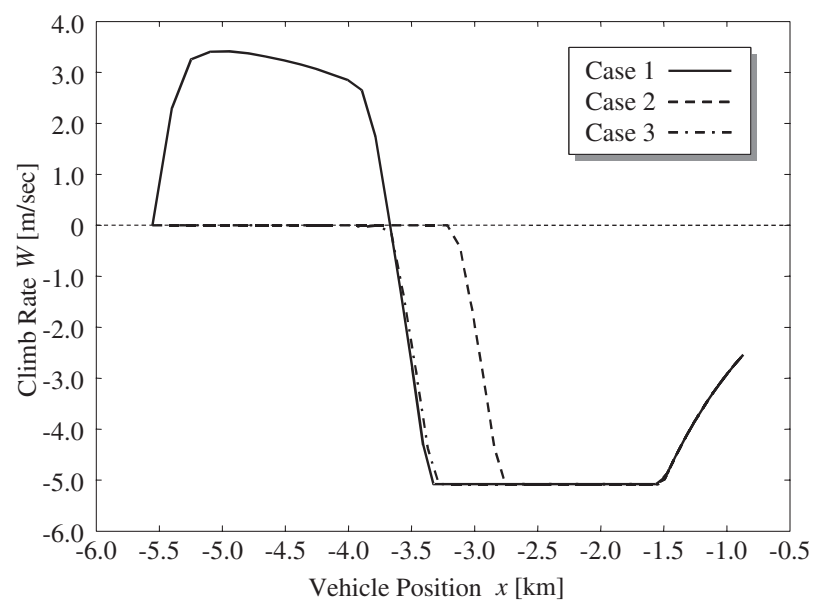

Fig. 5. Optimal solutions (climb rate history).

\subsection{Computational optimization results}

The results of the computational optimization are shown in Figs. 3-9 and Table 2. The noise model used in the study is largest when the flight path angle is in the range $-15 \mathrm{deg}$ to $5 \mathrm{deg}$, with its maximum at $-5 \mathrm{deg}$. Optimal solutions therefore attempt to avoid this flight path angle, and minimize the flight time as much as possible.

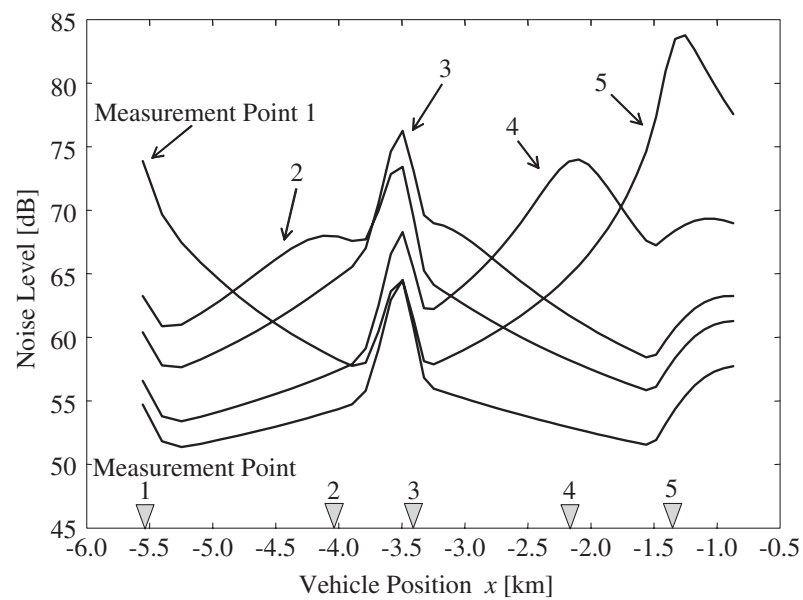

Fig. 6. Noise levels according to the optimal solution and position of the measurement points (Case 1)

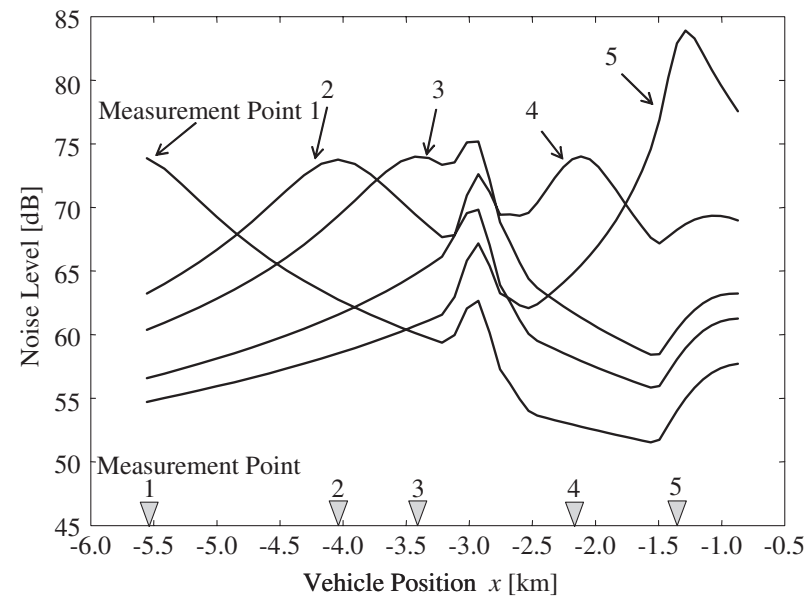

Fig. 7. Noise levels according to the optimal solution and position of the measurement points (Case 2).

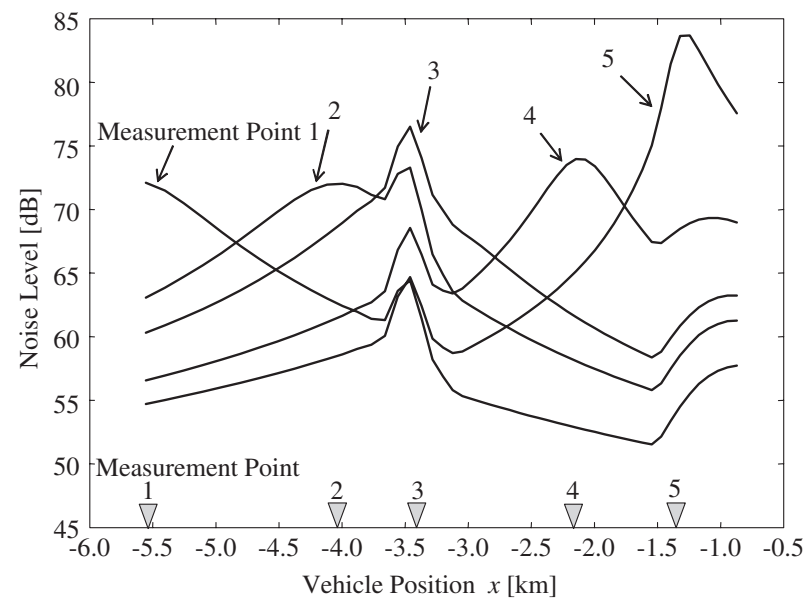

Fig. 8. Noise levels according to the optimal solution and position of the measurement points (Case 3 ).

In the problem setting of Case 1 (without wind, $u_{\mathrm{g}}=0$ ), a descent flight from the initial location towards the target point would be necessary to fly at a flight path angle that increases the noise. For this reason, in the optimal solution, there is a moment of ascent after the flight begins. The flight 


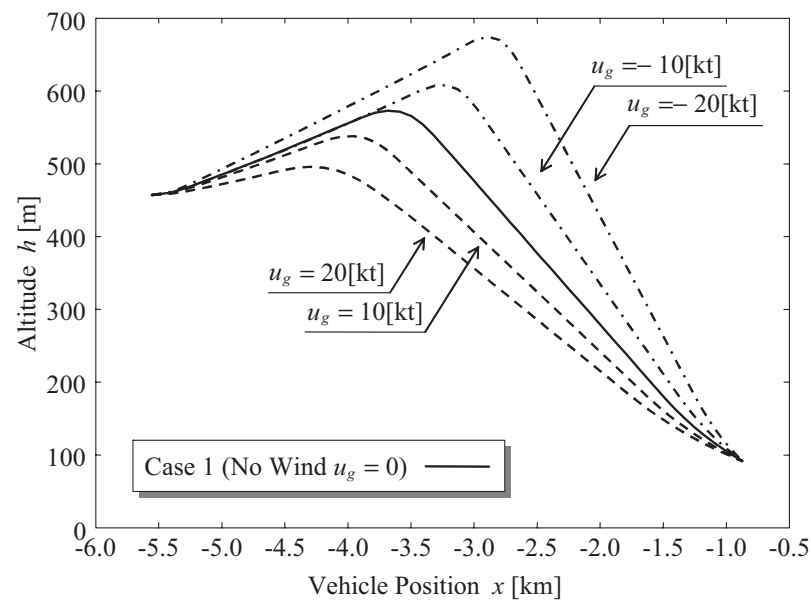

Fig. 9. Influence of wind on the optimal altitude history.

path angle at this time is about $4 \mathrm{deg}$. In order to reduce the flight time, the helicopter ascends at the flight path angle slightly less than the minimal noise angle of $5 \mathrm{deg}$. Upon quickly reaching an altitude of approximately $1850 \mathrm{ft}$, the helicopter starts to descend at the maximum descent rate $(W=-1000[\mathrm{fpm}])$. At this time, in order to avoid loud noise, the flight path angle passes through the vicinity of $-5 \mathrm{deg}$ as quickly as possible and ascent changes to descent. If the descent rate is $1000 \mathrm{fpm}$ and horizontal velocity is $100 \mathrm{kt}$ when the flight begins, the flight path angle would be $-5.6 \mathrm{deg}$, and a loud noise would occur. Thus, the helicopter decreases the horizontal velocity to the minimum velocity of $50 \mathrm{kt}$, and then descends at a flight path angle of $-11.2 \mathrm{deg}$. This is the descending flight path angle with the lowest noise within the permitted flight range. The flight changes from ascent to descent when the descending flight leads straight to the terminal point at the terminal altitude.

In Case 2 (without wind), in order to obtain a similar flight path to a normal approach, we conduct the computational optimization with a constraint condition preventing ascent flight. A horizontal velocity of $100 \mathrm{kt}$ is kept after the flight starts, but is soon reduced, and the maximum descent flight begins at a horizontal velocity of $50 \mathrm{kt}$. Since the flight time is reduced, the helicopter does not decelerate as soon as the flight begins. The descent trajectory is exactly the same as in Case 1 (without wind). In Case 3 (without wind), the initial altitude is $1850 \mathrm{ft}$, which is the maximum altitude in Case 1, and the constraint conditions are set to prevent ascent. We thought it would be better to start the flight at $1850 \mathrm{ft}$. Case 3 (without wind) shows, after flying horizontally, the helicopter makes the same descent flight as Case 1 (without wind).

Figures 6-8 indicate noise levels at measurement points for Cases 1-3 (without wind). When the descending helicopter takes up flight path angles yielding loud noise, and the flight path is shallow at a low altitude near the terminal time, the measured noises increase. In addition, when the helicopter passes over each measurement point, a loud noise is also measured. The optimal solutions show that the influence of the flight altitude on the noise is not particularly large in this study, but rather the influence of the flight path angle is dominant.

Table 2, which also includes the results of the flight experiments described later, shows the energy-based noise estimation (the performance index) $E_{\mathrm{A}}$, flight time $t_{\mathrm{f}}$ [sec] and temporal-mean noise level $L_{\mathrm{A}}[\mathrm{dB}]$. This temporal-mean noise level is a digital expression of the value obtained by dividing the estimation function calculated by time-integrating the noise energy (the energy-based noise estimation $E_{\mathrm{A}}$ ) by the flight time. That is to say, it is calculated as:

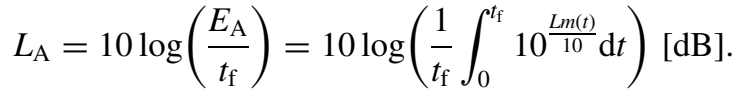

The table indicates that, though the momentary ascent in Case 1 causes its flight time to be longer than in Case 2, the amount of noise in Case 1 is smaller. The amount of noise in Case 1 is also smaller than in Case 3, whose initial altitude is higher. This means that the noise level has a higher dependence on the flight path angle rather than the flight altitude. However, the difference in the noise levels in these three cases is not particularly large.

Figure 9 shows the optimal flight paths for Case 1 with wind speeds $u_{\mathrm{g}}$ of $-20 \mathrm{kt},-10 \mathrm{kt}, 10 \mathrm{kt}$ and $20 \mathrm{kt}$. In each case, the characteristic descent following the ascent remains unchanged. The noise depends on the flight path angle calculated from airspeed rather than groundspeed. For example, with a headwind, the noise is reduced when the helicopter descends in a steep path. Thus, a difference in optimal flight path is yielded according to wind speed. The flight path angles calculated from the airspeeds are roughly the same in every case.

The optimal solutions obtained above differ from usual conventional approaches taken by pilots. Before conducting flight tests based on numerical optimization, we confirmed the tracking of the optimal solutions using a flight simulator.

Table 2. Comparison of optimal solutions and flight experiment results.

\begin{tabular}{|c|c|c|c|c|c|c|}
\hline & \multicolumn{2}{|c|}{$\begin{array}{l}\text { Performance index } \\
E_{\mathrm{A}} \\
\end{array}$} & \multicolumn{2}{|c|}{$\begin{array}{c}\text { Flight time } \\
t_{\mathrm{f}}[\mathrm{sec}]\end{array}$} & \multicolumn{2}{|c|}{$\begin{array}{l}\text { Temporal-mean noise level } \\
\qquad L_{\mathrm{A}}[\mathrm{dB}]\end{array}$} \\
\hline & $\begin{array}{l}\text { Optimal } \\
\text { solution }\end{array}$ & $\begin{array}{c}\text { Flight } \\
\text { experiment }\end{array}$ & $\begin{array}{l}\text { Optimal } \\
\text { solution }\end{array}$ & $\begin{array}{c}\text { Flight } \\
\text { experiment }\end{array}$ & $\begin{array}{l}\text { Optimal } \\
\text { solution }\end{array}$ & $\begin{array}{l}\text { Flight } \\
\text { experiment }\end{array}$ \\
\hline Case 1 & $3.02 \times 10^{8}$ & $3.76 \times 10^{7}$ & 149.1 & 124.2 & 63.1 & 54.8 \\
\hline Case 2 & $3.77 \times 10^{8}$ & $3.18 \times 10^{7}$ & 134.5 & 110.4 & 64.5 & 54.6 \\
\hline Case 3 & $3.57 \times 10^{8}$ & $3.79 \times 10^{7}$ & 145.9 & 115.9 & 63.9 & 55.1 \\
\hline Ordinary flight & & $4.38 \times 10^{7}$ & & 93.3 & & 56.7 \\
\hline
\end{tabular}




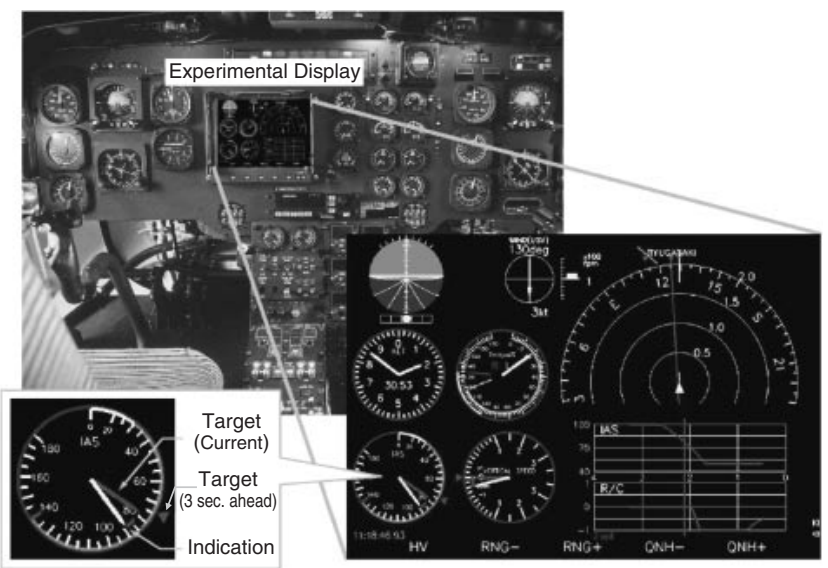

Fig. 10. Experimental display panel.

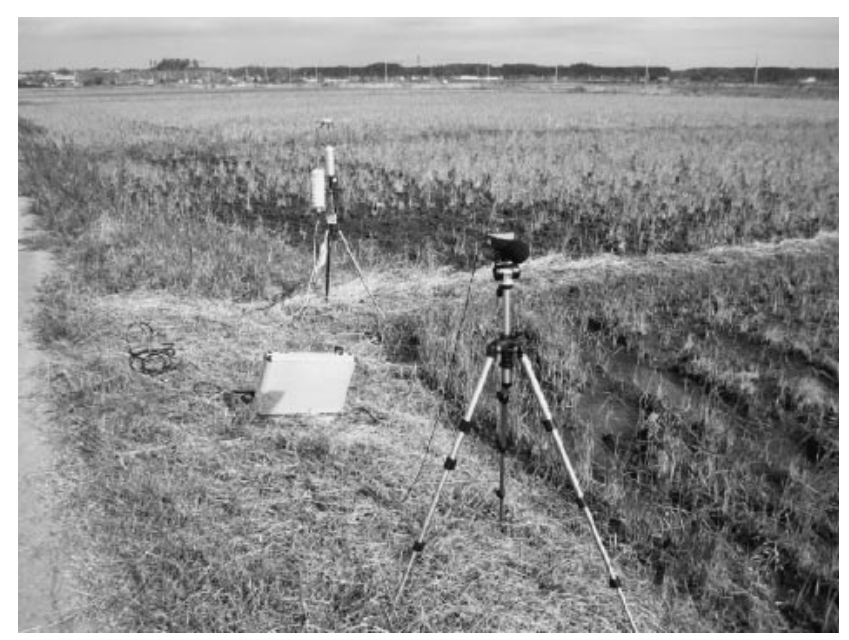

Fig. 11. Noise measurement device.

As a result, we obtained a comment from a pilot that adequate acquaintance with the timing of the altitude change made it possible to track the optimal solutions.

\section{Flight Experiments}

\subsection{Flight experiment method}

As stated above, we performed the flight experiments at Ryugasaki Airfield using a JAXA-owned MuPAL- $\varepsilon$ in order to confirm the optimal control results. All flights were conducted under the manual control of the pilot. In order to facilitate the tracking of the optimal solution, the flight conditions were indicated using an experimental display (Fig. 10) ${ }^{10)}$ installed on the instrument panel. In addition to the usual indicators for speed, climb rate and altitude, the flight conditions of the optimal solution 3 seconds ahead of the current time are also shown. Noise data was compiled at each of the five measurement points on the ground using noise measurement devices, as shown in Fig. 11. The flight experiments were conducted between November 30 and December 7, 2004. Since the wind at this time was weak and its influence was considered small, the optimal solution with $u_{\mathrm{g}}=0$ was used as the flight tracking objective. The optimal

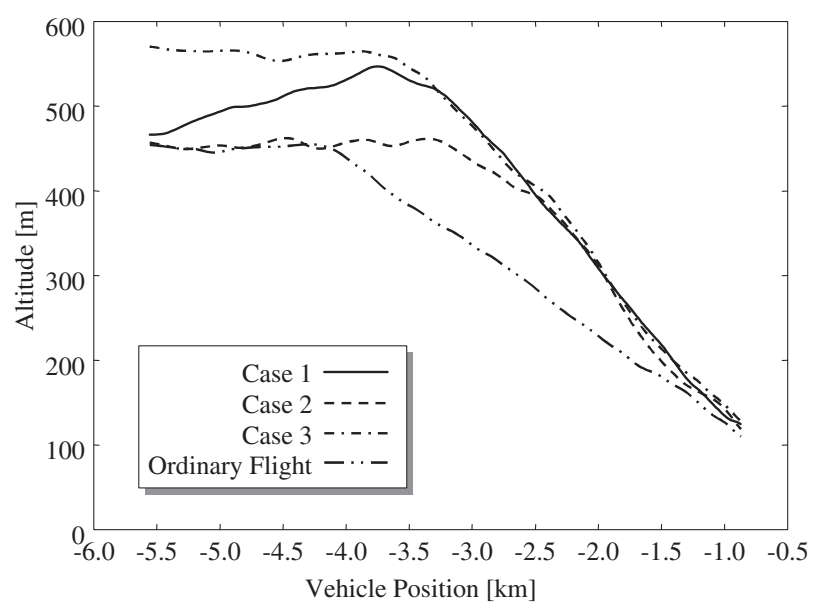

Fig. 12. Flight experiment (altitude history).

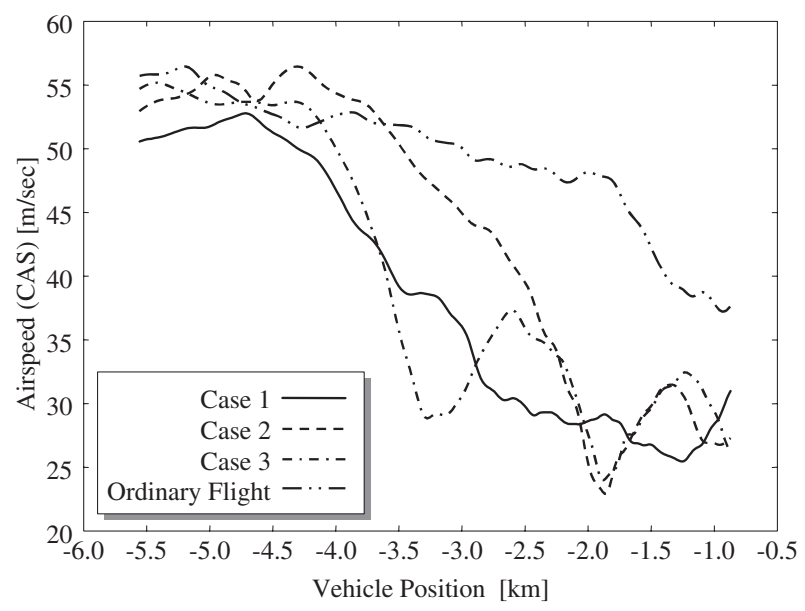

Fig. 13. Flight experiment (airspeed history).

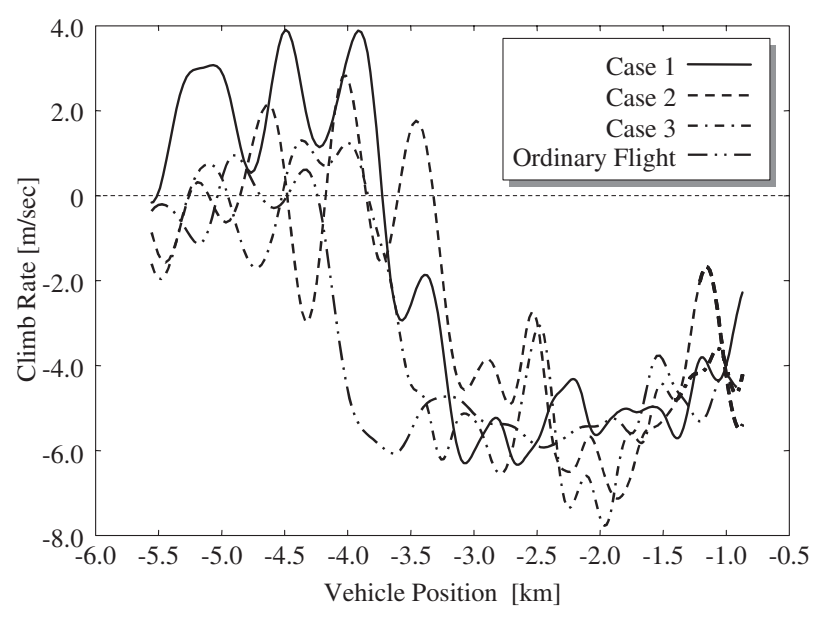

Fig. 14. Flight experiment (climb rate history).

solution with $u_{\mathrm{g}}=0$ and the results of the flight experiments are compared below.

\subsection{Flight experiment results}

The flight data obtained using the MuPAL- $\varepsilon$ 's measurement systems for each flight case are shown in Figs. 1214. A comparison of these with Figs. 3-5 results in that, since the overall tracking of the optimal solutions is precise, 


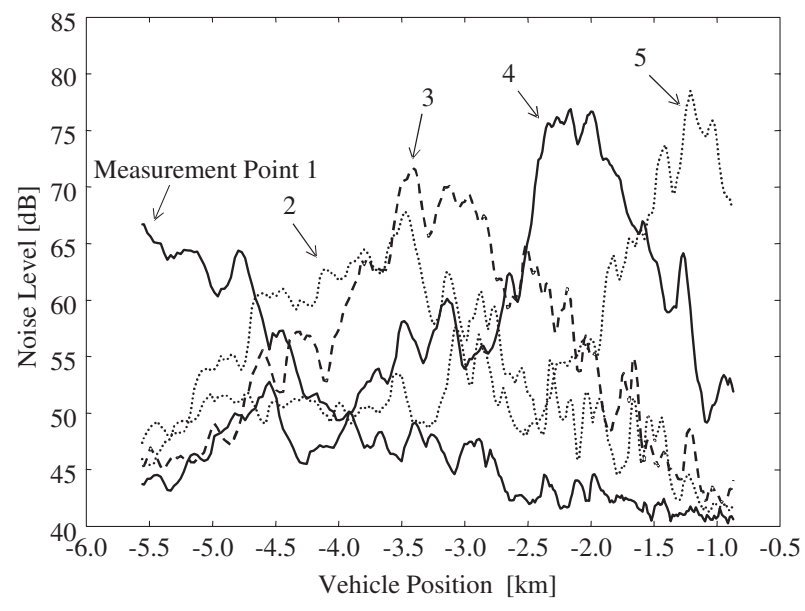

Fig. 15. Noise levels according to the flight experiment (Case 1).

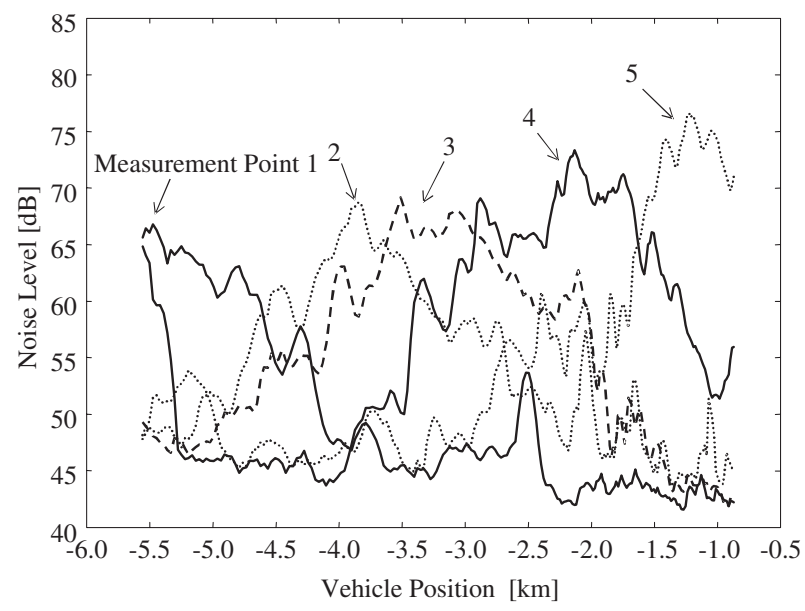

Fig. 16. Noise levels according to the flight experiment (Case 2).

the constraint conditions of the computational optimization shown in Eqs. (13)-(18) are defined appropriately. The airspeed in the experiments was faster than in the optimal solution, which is especially prominent after the transition from ascent to descent. While the climb rate is well controlled, there is oscillation within a range of about $2 \mathrm{~m} / \mathrm{sec}$. For computational optimization in the future, it is probably necessary to solve an optimal solution (a robust optimal solution) that suppresses additional noise due to tracking errors.

A landing approach that the pilot judged to be usual was also conducted in the flight experiments in order to compare it with the optimal flights. An example is indicated by "ordinary flight" in Figs. 12-14. In the ordinary landing approach flight, the helicopter performs horizontal flight close to the runway, and then descends at a relatively steep angle. It usually decelerates near the runway.

The noise levels actually recorded at the measurement points are shown for the four flight cases in Figs. 15-17. These overall trends are well matched with those of the noise levels predicted by the optimal solutions in Figs. 68 , but there is a maximum error of around $10 \mathrm{~dB}$ at each measurement point. We overestimated the noise level in the optimal solution. This is probably attributed to underes-

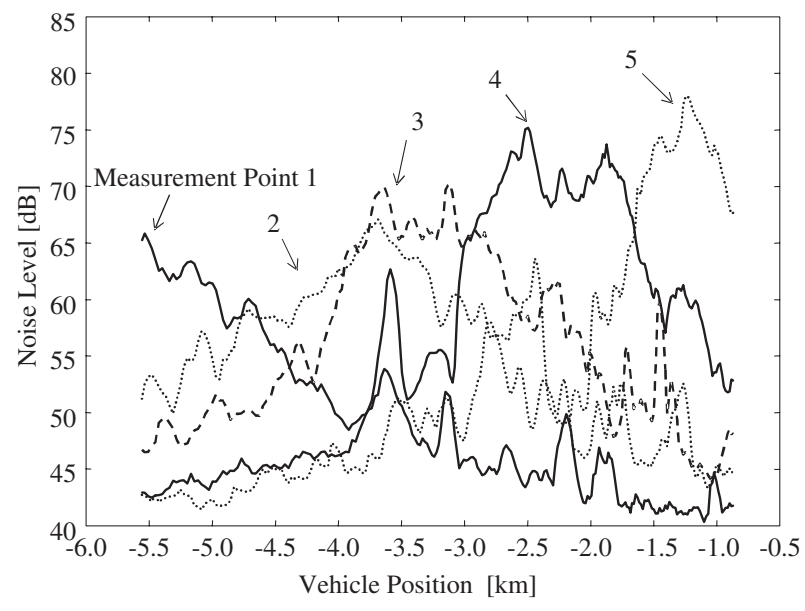

Fig. 17. Noise levels according to the flight experiment (Case 3).

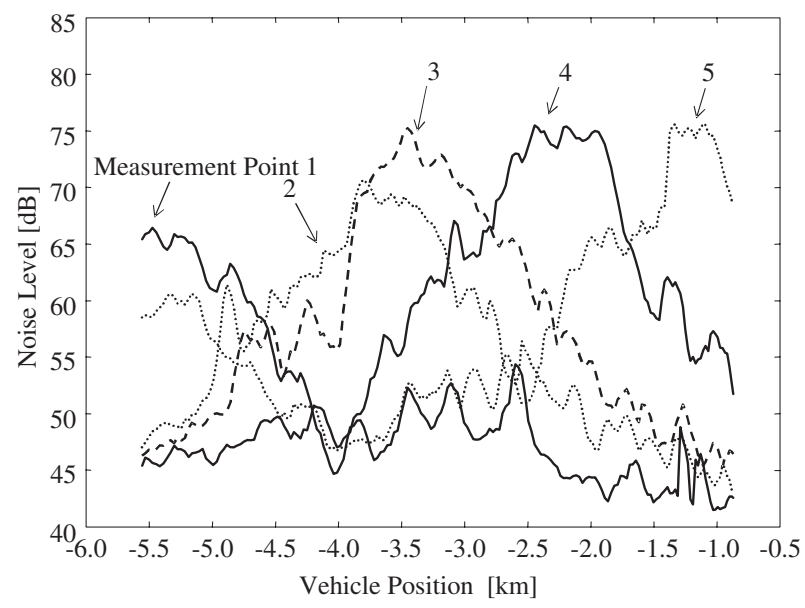

Fig. 18. Noise levels according to the flight experiment (ordinary flight).

timating the noise attenuation, which is based only on the inverse square law of distance.

Table 2 summarizes the results of the flight experiments and the optimal solutions. The flight time is shorter in the experiments than in the optimal solutions. This suggests that, although it was judged that there was no wind at the time of conducting the experiments, a slight tailwind may in fact have been blowing. The values of the performance indexes are lower by a factor of 10 in the flight experiments than in the optimal solutions. The temporal-mean noise levels are therefore also $10 \mathrm{~dB}$ lower in the results of the flight experiments. This is due to the problem of the attenuation model, as previously stated. In the flight experiments, the difference in the noise levels for the three cases is small, and in contrast to the optimal solution, the noise level for Case 1 is not the smallest. In the future it will be necessary to use more precise sound-source models with directivity etc., and more precise sound propagation models including fluctuations due to such factors as wind and humidity. It should be noted that, while the flights according to the optimal solutions have longer flight times than the ordinary landing approach flight, reducing the noise level by as much as $2 \mathrm{~dB}$ is successfully achieved. 


\section{Conclusions}

This study solved the flight profiles of the helicopter landing approach that minimizes the ground-level noise using the computational optimization. We obtained the flight that is characterized by a short flight time and a flight path angle that suppresses BVI noise. Furthermore, the flight experiments following the optimal solutions confirmed that the noise could be decreased in comparison with the conventional flight.

Since this is our first study, we dealt with the simplified sound source and propagation models, and the two-dimensional helicopter motion model limited to the vertical plane. At present, we are investigating the refinement of each model for higher precision, and methods for obtaining an optimal solution robust to tracking errors. Furthermore, in the future, in order to expand to optimization with a threedimensional helicopter motion model, we will investigate optimization methods and constraint conditions for lateral motion, and address the noise minimization problem based on land utilization.

\section{References}

1) Funabiki, K., Muraoka, K., Iijima, T. and Shiomi, K.: NOCTARN: Trajectory Based CNS/ATM Concept for Small Aircraft, Proceedings of 21st Digital Avionics Systems Conference, 2002, pp. 485-492.

2) Ishii, H., Gomi, H., Okuno, Y., Uchida, J. and Tsuchiya, T.: Flight Tests of Helicopter Noise Abatement Operations, 31st European Rotorcraft Forum, Paper \#28, 2005.

3) Ishii, H., Okuno, Y. and Funabiki, K.: Flight Experiments for Aircraft Noise Measurement Using “Tunnel-in-the-Sky” Display, AIAA Paper 2002-4880, 2002.

4) Ishii, H., Gomi, H. and Okuno, Y.: Helicopter Flight Tests for BVI Noise Measurement Using an Onboard External Microphone, AIAA Paper 2005-6119, 2005.

5) Ochi, A., Aoyama, T., Saito, S., Shima, E. and Yamakawa, E.: BVI Noise Predictions by Moving Overlapped Grid Method, AHS 55th Annual National Forum, 1999, pp. 1400-1413.

6) Tsuchiya, T. and Suzuki, S.: Computational Method of Optimal Control Problem Using Mathematical Programming (2nd Report) Introduction of Block Diagonal Hessian Method, J. Jpn. Soc. Aeronaut. Space Sci., 46 (1998), pp. 497-503 (in Japanese).

7) Lucas, M.J. and Marcolini, M.A.: Rotorcraft Noise Model, American Helicopter Society Technical Specialists' Meeting for Rotorcraft Acoustics and Aerodynamics, Williamsburg, VA, 1997.

8) Ishii, K., Gomi, H. and Okuno, Y.: Acoustic Flight Tests Using MuPAL- $\varepsilon$ Research Helicopter, Proceeding of the 42nd Aircraft Symposium, 2004, pp. 79-86 (in Japanese).

9) Tachibana, H.: Energy-Based Noise Estimation, Aircraft Environment Research, 7 (2003), pp. 3-11 (in Japanese).

10) Funabiki, K.: Design of Tunnel-in-the-Sky Display and Curved Trajectory, 24th International Congress of the Aeronautical Science, Yokohama, Japan, 2004. 